\title{
Portable Medical Equipment Holder Assists Pediatric Venipuncture
}

\author{
Eugen-Matthias Strehle ${ }^{*, 1}$ and Thomas William Needham ${ }^{2}$
}

\author{
${ }^{I}$ North Tyneside General Hospital, North Shields NE29 8NH, UK \\ ${ }^{2}$ Medical School, Newcastle University, Newcastle Upon Tyne NE2 4HH, UK
}

\begin{abstract}
Objectives: Phlebotomy and venous cannulation are common, technically challenging and potentially distressing procedures in pediatrics. The aim of this pilot study was to assess whether a novel medical equipment holder could be a useful adjunct for venipuncture in children.

Methods: Doctors and nurses at a district general hospital were asked to analyze the portable medical equipment holder and complete a questionnaire. The questionnaire consisted of twelve questions regarding seniority of the practitioner and their opinions of the device.

Results: Fifty-three questionnaires were completed by a variety of pediatric health professionals. Overall, 98\% of participants rated the device as useful, $2 \%$ assumed a neutral position and no practitioner described the device as not useful. A positive first impression was shared by $92 \%$ of the participants. A non-disposable device was preferred over the disposable alternative. The square shape and plain colour of the prototype were considered suitable by the majority of participants. Plastic was the preferred material. The modal price range suggested for a non-disposable device was $£ 5$ ( $\$ 8$ ) or less. Several modifications to the design were suggested.

Conclusion: The portable medical equipment holder was well received by the pediatric staff. Design modifications put forward by the participants progressed to the development of an improved device. Further clinical research, preferably in a multi-centre study, is required.
\end{abstract}

Keywords: Blood container, blood tube, capillary tube, medical equipment holder, phlebotomy, portable holder, venipuncture, venous cannulation, venous catheterization.

\section{INTRODUCTION}

Venipuncture is the most common invasive procedure in pediatrics but is widely regarded as challenging for the practitioner and traumatic for the young child [1, 2]. Targeting smaller veins, using smaller equipment and the patient's fear and pain are all contributing factors. The potential difficulty and time consuming nature of the procedure were recently demonstrated by Larsen et al. who found that gaining peripheral intravenous access in children takes on average 28 minutes and two venipuncture attempts [3]. This combination of difficulty and the emotional and physical distress of the patient explain why research is extensive and continuous in an attempt to improve the procedure. Several studies have concentrated on improving the child's experience of the procedure such as the search for the optimal form of analgesia, including the use of a hand held laser device which improves anaesthetic potency, and the use of psychological distraction techniques [4-8]. Hands et al. found that despite the widespread knowledge of the psychological distress and pain generated by venipuncture, more could be done before and during the procedure to alleviate this [9]. Minimising the distress for the child during venipuncture is of vital importance to prevent the generation

*Address correspondence to this author at the North Tyneside General Hospital, North Shields NE29 8NH, UK; Tel: 0044191 2031200;

Fax:00441912932520; E-mail: strehle@doctors.org.uk of needle phobia, an affliction that can remain with the patient for many years. Technological advances in the equipment used during the procedure have also been the subject of study. Analyses of novel deviceswhich help the practitioner to locate small and inaccessible veins using near infrared light have yielded positive results and routine, clinical application of these devices could significantly improve the procedure [1, 10-12]. The depth of research into this subject area is illustrated by a study which evaluated the best dressing for securing peripheral intravenous cannulae [13]. However, these avenues of research fail to address two significant problems associated with the procedure, namely needle-stick injuries and blood spillages. Research from a German university hospital showed that the prevalence of avoidable needle-stick injuries was particularly high within the pediatric department [14]. It is also widely accepted among health professionals that blood spillage is a common problem during venipuncture in children. Despite this, there is no evidence of research reporting a device which facilitates the safe storage of pediatric blood containers and needles during the procedure. The aim of this pilot study was to evaluate a novel medical equipment holder specific to pediatric practice which could be a useful adjunct for venipuncture.

\section{METHODS}

The study was carried out in the pediatric department of a district general hospital between the $1^{\text {st }}$ January and the $31^{\text {st }}$ March 2011. The department assesses and treats children 


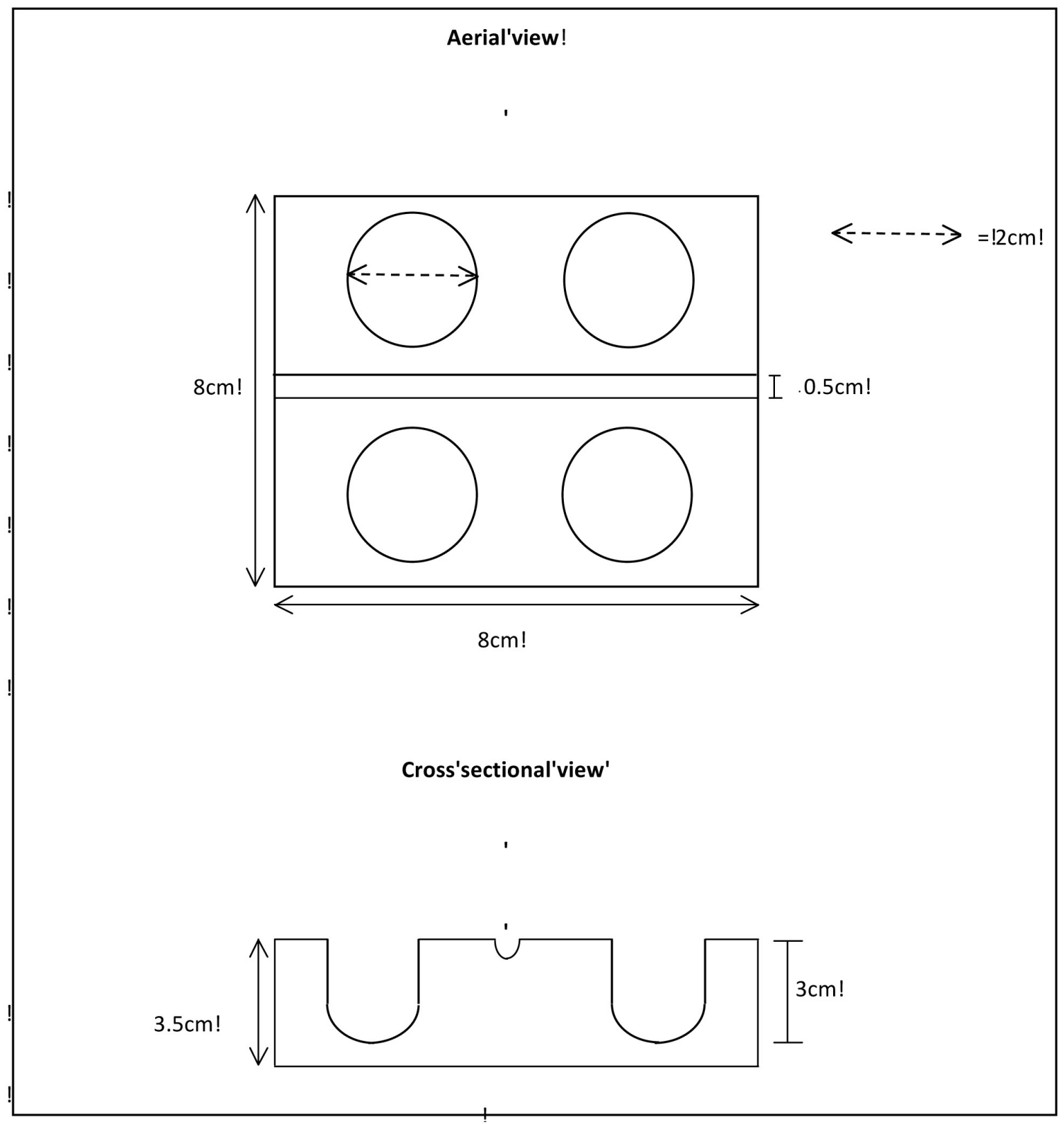

Fig. (1). Diagrammatic representation of the portable medical equipment holder.

from 0 to 16 years of age. The study aimed to evaluate a novel piece of apparatus which could be a useful adjunct for venipuncture. Pediatric doctors and nurses were asked to closely examine the device and then complete a questionnaire. The questionnaire contained 12 open and closed questions concerning role of the practitioner during venipuncture and their opinions of the device. The device is specifically designed to hold equipment during pediatric phlebotomy and venous cannulation. The prototype device used in this study was made of hardwood and coloured by white waterproof paint. The device was cuboidal in shape and measured $8 \mathrm{~cm} \times 8 \mathrm{~cm} \times 3.5 \mathrm{~cm}$. The four identical bores were $2 \mathrm{~cm}$ in diameter and $3 \mathrm{~cm}$ deep, designed to hold pediatric blood containers. The central recess was $0.5 \mathrm{~cm}$ wide and $0.2 \mathrm{~cm}$ deep, suitable for storing an injection needle or a capillary tube (Fig. $\mathbf{1})$.

\section{RESULTS}

Fifty-three questionnaires were completed by the following groups of pediatric health professionals: 6 consultants, 5 registrars, 1 associate specialist, 1 staff grade doctor, 13 senior house officers, 3 advanced nurse practitioners, 2 specialist nurses, 2 sisters, 11 staff nurses, 6 auxiliary nurses, 2 students and 1 play coordinator. Practitioners who directly performed the procedure made up $66 \%$ of the cohort, $30 \%$ assisted with the procedure and the remaining $4 \%$ observed the procedure. Overall, $98 \%$ of 
participants rated the device as useful and $2 \%$ assumed a neutral position in terms of its usefulness. No participant in the study rated the device as not useful (Table 1). Among the qualified practitioners $(n=51)$, the mean amount of experience post qualification was 8.2 years (98 months, range 6 to 336 months). Students accounted for the other two responses.

Table 1. Role of health professional during venipuncture procedures and overall rating of the device.

\begin{tabular}{|c|c|c|c|}
\hline Role & Useful (\%) & Neutral (\%) & Not Useful (\%) \\
\hline \hline Performing(n=35) & 100 & 0 & 0 \\
Assisting(n=16) & 94 & 6 & 0 \\
Observing(n=2) & 100 & 0 & 0 \\
\hline
\end{tabular}

The participants were asked to document their first impressions of the device. The majority of the responses were positive with $45 \%$ believing it to be a good idea and $38 \%$ describing it as very good or excellent. A further $9 \%$ believed the device was useful or practical. The negative responses $(8 \%)$ were provided by individuals who had seen or used a similar piece of apparatus previously. A nondisposable form of the device was favoured by $47 \%$ of participants. Conversely, 34\% preferred a disposable model. Of the remaining individuals, $13 \%$ wanted the device to be available in both forms and $6 \%$ demonstrated no preference between the two options. A plain colour such as white was preferred by $42 \%, 30 \%$ said that the colour did not matter and $19 \%$ opted for a bright colour, for example yellow. Four individuals $(8 \%)$ commented that child friendly pictures or patterns should be present. The one remaining participant didn't quote a colour in their response but expressed a desire for colour differentiation between disposable and nondisposable forms. A variety of materials were suggested for the device: plastic $(83.0 \%)$, dense foam $(5.7 \%)$, metal $(5.7 \%)$, cardboard $(3.8 \%)$ and rubber $(1.9 \%)$. The majority of participants $(81.1 \%)$ concurred that the square shape of the prototype was ideal for the device. Other shapes suggested were: circular $(9.4 \%)$, rectangular $(5.7 \%)$ and oblong $(1.9 \%)$. The remaining participant $(1.9 \%)$ said that the shape of the device did not matter. Widely varying values were suggested as the recommended cost of the device depending on whether it was in a disposable or nondisposable form. In a disposable form, the valuations provided were: $10 \mathrm{p}(\$ 0.2)$ or less $(n=12)$, between $10 \mathrm{p}(\$ 0.2)$ and 50p $(\$ 0.8)(n=2)$, from 50p $(\$ 0.8)$ to $£ 1(\$ 1.6)(n=13)$, between $£ 1(\$ 1.6)$ and $£ 5(\$ 8)(n=2)$ and more than $£ 5(\$ 8)$ $(n=1)$. In a non-disposable form, the recommended costs were: $£ 5(\$ 8)$ or less $(n=30)$, between $£ 5(\$ 8)$ and $£ 10(\$ 16)$ $(n=3)$, from $£ 10(\$ 16)$ to $£ 30(\$ 47)(n=15)$ and more than $£ 30$ (\$47) $(n=1)$. The figures in these respective categories do not summate to fifty-three because some participants stated a cost for both a disposable and non-disposable device whereas other participants chose to valuate either the disposable or the non-disposable form. The prototype device used for this study contained four bores and one recess. The majority of participants (64\%) agreed that four bores was a suitable number. On the other hand, $32 \%$ wanted more than four bores and the remaining 4\% wanted less than four bores. Thirty-three participants $(62 \%)$ shared agreement that one recess was correct. Fifteen individuals (28\%) wanted an extra recess added to the design and two people (4\%) wanted more than two recesses. Three responders $(6 \%)$ did not comment on the number of recesses.

Finally, the participants were asked to suggest any other modifications that could be made to the device. Eleven individuals deemed no further improvements necessary (21\%). The most frequent recommendations were: adding a tray to the device $(n=8)$, making space available for the storage of lids $(n=6)$, making the model smaller $(n=9)$ and making the model lighter $(n=6)$. Other suggestions included adding a handle to the design $(n=2)$, making the bores deeper and narrower to ensure tighter fitting of tubes $(n=4)$, making the recess deeper $(n=1)$ and adding a stand to the design $(n=1)$. Two individuals expressed that they found the prototype device slippery and that a new material should be used to prevent this. One response stated that the devices should be designed so that they could be stacked upon each other and another stressed the importance of using a material that was easily washable.

\section{DISCUSSION}

In this single-centre study, fifty-three pediatric health professionals were asked to examine and comment on a novel device which could be a useful piece of apparatus during venipuncture in children. The portable medical equipment holder was well received by the pediatric staff. The vast majority of participants rated the device as useful. Both degree of seniority and role during the procedure had no significant influence on the responses provided. In terms of their first impression, eighty-three per cent described the device as good or excellent. The simplicity of the idea should not detract from its potential benefit as an adjunct for venipuncture. Evidence states that phlebotomy is a complex fine motor skill, even for the experienced practitioner, that requires good hand-eye coordination, manual dexterity and regular practice $[7,15]$. Despite these findings, the senior practitioners in our study were no less receptive to the device than their junior colleagues. The usefulness of the device arises from its ability to hold open and closed pediatric blood containers in a secure, upright position which enables the simple deposition of blood into the container and the organised storage of equipment during the procedure. In clinical practice, the device can literally provide the proverbial 'extra pair of hands' and may improve the efficiency of the procedure and reduce the number of blood spillages. The recess also facilitates the safe storage of an injection needle and could reduce the incidence of avoidable needle-stick injury, a recognised risk of pediatric venipuncture [14].

The majority of the questionnaire focused on specific design issues including the preferred colour, shape and material for the device. These responses provided the foundations for some modifications and the development of a new design. The modified device is circular in shape, contains six bores and two recesses and is white in colour. A storyboard reveals the new design and depicts the device in clinical practice (Fig. 2). The extra number of bores and recesses allows more storage space for blood containers, injection needles and capillary tubes. Several participants suggested adding a removable tray to the device. This could 


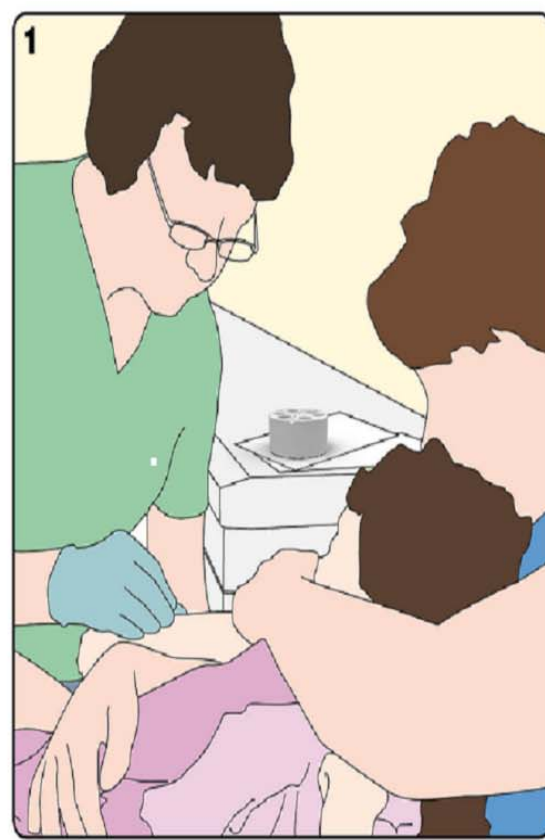

Taking a blood sample from a small child is not often easy, often requiring a second nurse or parent to assist by holding the child The doctor takes a sample using a needle and syringe.

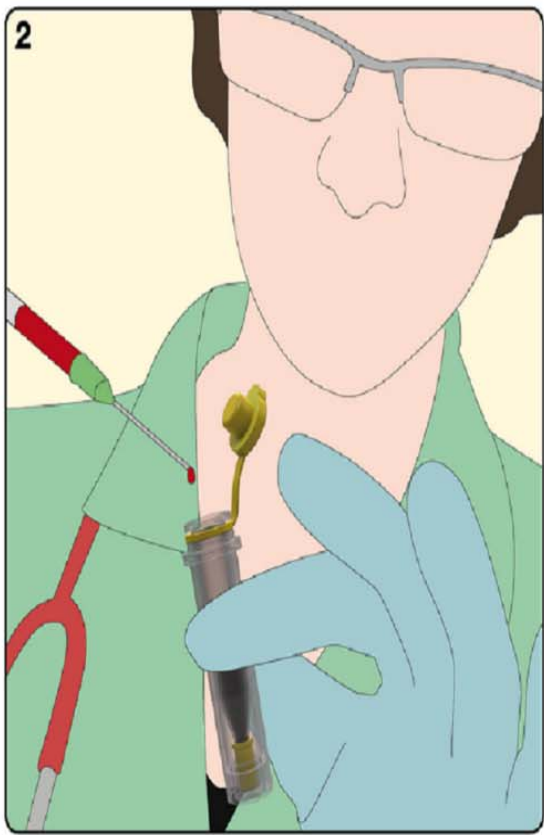

Once the blood sample has been taken, the doctor or nurse places the blood sample into a vial.

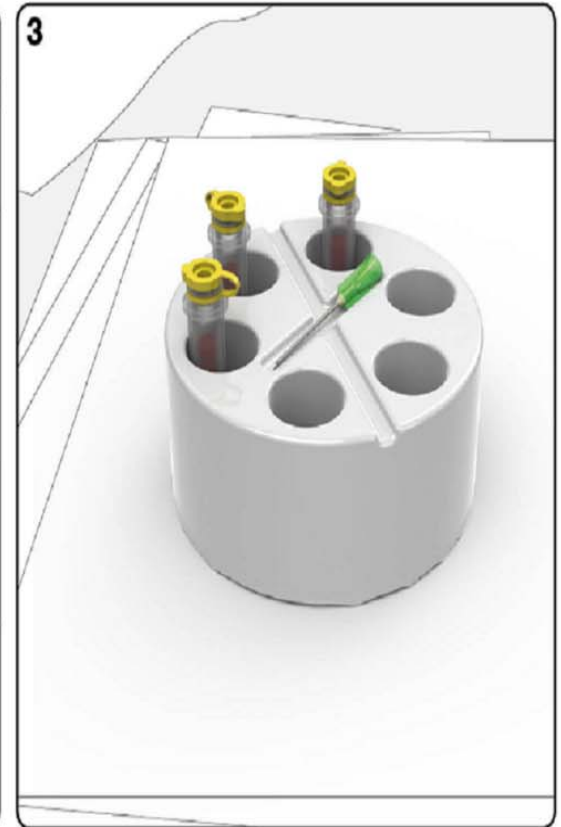

The doctor or nurse can place the vials in the blood container. Quickly returning to the child safe in the knowledge that the vials are safe and secure.

Fig. (2). Cartoon illustrating the use of the portable medical equipment holder during a pediatric venipuncture: A Taking blood from the child B Injecting blood into a pediatric blood tube. C Storing blood tubes and injection needle in the portable equipment holder.

facilitate the storage of other small pieces of equipment used during venipuncture such as plasters, cotton wool buds, gauzes, gloves and alcohol wipes. A non-disposable form of the device was favoured over the disposable alternative. Each option has its own respective strengths and weaknesses. A disposable device could be hygienically disposed of at the bedside and would be very cheap to produce. The modal cost suggested by the participants for a disposable device was in the range of 50 pence $(\$ 0.8)$ to $£ 1$ (\$1.6). A non-disposable device would have to be cleaned on a regular basis and should be made stackable to reduce the required storage space. It is also worth taking into account the possibility of losing the device or it being temporarily taken by other health professionals which could be an inconvenience for the practitioner. If the device was to become a routine piece of apparatus for pediatric venipuncture, the non-disposable form would be the more financially viable option. The modal price suggested for a non-disposable device $£ 5(\$ 8)$ or less. The costs suggested ranged from $£ 3(\$ 5)$ to $£ 35(\$ 55)$. If the device were to become routinely used in clinical practice, the production costs should be minimal to facilitate mass production and an affordable retail price. The participants considered plastic as the ideal material for the device. Plastic does appear to be a suitable choice because it is inexpensive to produce, easily cleaned and recyclable.

Four individuals commented that they had used a different piece of apparatus with a similar function in their previous practice. However, these were makeshift adaptations of simple test tube racks designed to hold adult sized tubes and did not possess a recess or the pediatric specificity which makes this device unique. An internet search revealed that similar pieces of apparatus are commercially available. However, these products are also designed to hold adult sized tubes and vacutainers which are not used in pediatric practice. There is also no evidence of a pediatric blood tube and injection needle holder subjected to clinical testing. This emphasizes the novelty of the device demonstrated in this study. Clinical application of the portable medical equipment holder could reduce the time required for phlebotomy and venous cannulation whilst also reducing the requirement for repeat venipunctures by preventing blood spillages. These interventions would significantly improve procedural efficiency and diminish the psychological and physical distress for the young patient involved. Future research should involve in-depth clinical analysis of the modified device across multiple centres.

\section{AUTHORS CONTRIBUTION}

EMS has designed the study, conducted the survey and co-wrote the manuscript. TWN evaluated the survey and cowrote the manuscript.
ABBREVIATIONS
$\% \quad=\quad$ Percentage
$\$=$ US Dollar
$£ \quad=\quad$ Pound Sterling
$\mathrm{n} \quad=\quad$ Number 


\section{CONFLICTS OF INTEREST}

EMS has been granted a patent for the device in the United Kingdom (GB2486291).

\section{ACKNOWLEDGEMENTS}

We would like to thank the pediatric medical and nursing staff at North Tyneside General Hospital who participated in the study.

\section{REFERENCES}

Strehle EM. Making the invisible visible: Near-infrared spectroscopy and phlebotomy in children. Telemed J E Health 2010; 16(8): 889-93

[2] Hands C, Round J, Thomas J. "When someone stabs you": Children's perspective of venepuncture. Arch Dis Child 2009; 94(6): 466.

[3] Larsen P, Eldridge D, Brinkley J, et al. Paediatric peripheral intravenous access: does nursing experience and competence really make a difference? J Infus Nurs 2010; 33(4): 226-35.

[4] Biran V, Gourrier E, Cimerman P, Walter-Nicolet E, Mitanchez D, Carbajal R. Analgesic effects of EMLA cream and oral sucrose during venipuncture in preterm infants. Pediatrics 2011; 128(1): e63-e70

[5] Gilboy S, Hollywood E. Helping to alleviate pain for children having venepuncture. Paediatr Nurs 2009; 21: 14-9.
[6] Zempsky W, Bean-Lijewski J, Kauffman R, et al. Needle-free powder lidocaine delivery system provides rapid effective analgesia for venipuncture or cannulation pain in children: Randomized, double-blind comparison of venipuncture and venous cannulation pain after fast-onset needle-free powder lidocaine or placebo treatment trial. Pediatrics 2008; 121(5): 979-87.

[7] Duff AJ. Incorporating psychological approaches into routine paediatric venepuncture. Arch Dis Child 2003; 88: 931-7.

[8] Singer AJ, Weeks R, Regev R. Laser assisted anaesthesia reduces the pain of venous cannulation in children and adults: a randomized controlled trial. Acad Emerg Med 2006; 13(6): 623-8.

[9] Hands C, Round J, Thomas J. Evaluating venepuncture practice on a general children's ward. Paediatr Nurs 2010; 22(2): 32-5.

[10] Strehle EM. Novel vein viewing system assists with venepuncture in children. J Pediatr Intensive Care 2012; 1: 1-2.

[11] Hess HA. A biomedical device to improve pediatric vascular access success. Pediatr Nurs 2010; 36(5): 259-63.

[12] Ernst DJ. Take the guesswork out of venipunctures. MLO Med Lab Obs 2009; 41(4): 18,20-21.

[13] Needham R, Strehle EM. Evaluation of dressings used with local anaesthetic cream and for peripheral venous cannulation. Paediatr Nurs 2008; 20(8): 34-6.

[14] Wicker S, Jung J, Allwinn R, Gottschalk R, Rabenau HF. Prevalence and prevention of needlestick injuries among health care workers in a German university hospital. Int Arch Occup Environ Health 2008; 81(3): 347-54

[15] Willock J, Richardson J, Brazier A, Powell C, Mitchell E. Peripheral venepuncture in infants and children. Nurs Stand 2004; 18: 43-50. 